\title{
Analytical Procedure Range
}

National Cancer Institute

\section{Source}

National Cancer Institute. Analytical Procedure Range. NCI Thesaurus. Code C134240.

The interval between the upper and lower concentration (amounts) of analyte in the sample (including these concentrations) for which it has been demonstrated that the analytical procedure has a suitable level of precision, accuracy and linearity. 